\title{
Das Buch mit sieben Siegeln. \\ Studien zum literarischen Aufbau der Offenbarung Johannis.
}

\author{
Von W. SattIer in Holzwickede.
}

\section{Das Gebet der Märtyrer und seine Erhörung ${ }^{1 .}$}

Nach dem Vorüberbrausen der an Sach I sff. erinnernden apokalyptischen Reiter steht vor dem Auge des Sehers mit der Öffnung des fünften Siegels (Apoc $6_{9-11}$ ) plötzlich ein ganz andersartiges

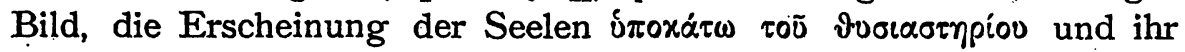
Rachegebet, - wiederum genau entsprechend dem weiteren Verlauf bei Sacharja ( I2ff. $_{\text {f. }}$; wo im unmittelbaren Anschlub an das Nachtgesicht von den Reitern im Myrtengrunde der Engel Jahwes spricht: iJahwe der Heerscharen, wie lange willst du dich nicht erbarmen Jerusalems und der Städte Judas, auf die du gezürnt hast diese siebenzig Jahre? Heil und Linderung der Not sagt Jahwe darauf dem angelus interpres zu; parallel zu seiner tatkräftigen Liebe gegen Jerusalem wird sich sein Zorn gegen die Heiden auswirken, $1_{14-17}$. Solange sich die Weltvölker ungestörten Friedens erfreuten, konnte Juda keine wesentliche Besserung seiner Lage erwarten; dennoch fehlte es nicht an Vorzeichen einer Verwirklichung der längst geweissagten Herrlichkeit, die für das aus Babel erlöste Volk anbrechen sollte.

Die vielfachen Berührungspunkte zwischen dem ersten Nachtgesicht des Sacharja und den fünf ersten Siegelvisionen Apoc 6 nach Seiten des Inhalts wie der Komposition liegen am Tage. Jedenfalls ist es schwer begreiflich, wie man die inmitten der unmittelbar folgenden furchtbaren Erdbebenvision Apoc $6_{12-17}$ einerseits und des unmittelbar vorher berichteten Auftretens der apokalyptischen Reiter anderseits eingeordnete Erscheinung der Seelen ó als einen Ruhepunkt (Weiß, Forschungen S. 7) hat bezeichnen können. Die Schrecken der Verfolgungszeit bilden den Hintergrund der Szene, die Seelen der Märtyrer sind wild bewegt durch die un-

1 Ich zitiere W. Boussets und H. Holtzmanns Erklärungen der Apokalypse nur mit den Namen der Verfasser, «Boll. Offb.c ist F. Boll, Aus der Offenbarung Johannis, 1914, JJ. Weiss Forschungenष ist Joh. Weiss, Die Offenbarung des Johannes, $1904=$ Forschungen z. Relig. u. Liter. d. A u. NT. Heft 3. 
ablässig lebendige Erinnerung an die Leiden jener Trübsalstage, in leidenschaftlicher Sehnsucht harren sie der Stunde, da ihr Blut an den Bewohnern der Erde heimgesucht werden soll, man hört ihr lautes Rachegeschrei. Eine solche Szene ist, schon an sich betrachtet, kein Ruhepunkt, und auf den Fortschritt der Handlung gesehen, trifft die Bezeichnung womöglich noch weniger zu. Es läßt sich im Gegenteil zeigen, daß ganz analog der Schilderung bei Sacharja diese Szene innerhalb des dramatischen Aufbaus unserer Apoc ein erregendes Moment von besonderer Wichtigkeit darstellt.

Sehr unvermittelt, was innerhalb der A poc allerdings nicht sonderlich überraschen kann, wird "als etwas ganz Selbstverständliches" (Bousset 270) $6_{9}$ ein (wie $8_{3}$ ) mit dem bestimmten Artikel bezeichneter, die Existenz eines himmlischen Tempels (vgl. I I 19 I 5 s. 715 I6 1) voraussetzender A1tar erwähnt, von dem vorher nichts gesagt ist. $\mathrm{Ob}$ dieser doch nicht ohne weiteres mit dem. Thron Gottes (Bousset 273f.) zu identifizierende Altar auch dem Seher erst jetzt erscheint, oder ob dieser ihn schon früher gesehen und als für den Gang der Handlung vorerst noch entbehrlich bloß unerwähnt gelassen hat, ist für den Gang unserer Untersuchung belanglos; auch $\sigma_{9}$ wird der Altar nicht um seiner selbst willen genannt: es kommt vielmehr auf die Seelen an, die der Apok. ijoxátw

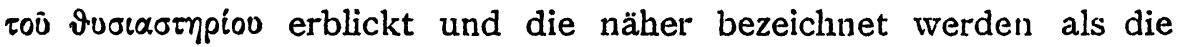
Seelen der um des Wortes Gottes und um des Zeugnisses willen, das sie hatten, Geschlachteten ${ }^{1}$.

Über die seit dem Tode der Märtyrer bereits verflossene Warte-

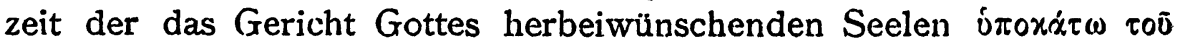
$\vartheta 0 \sigma$ `ornpiou läßt sich ebensowenig etwas Genaueres aussagen wie über die vom Apok. erblickte Anzahl der Seelen. Warum hier lediglich auf die Neronischen Christenmorde angespielt und von Kleinasien als Verfolgungsstätte geradezu abzusehen sein soll ${ }^{2}$, hat bisher niemand ausreichend begründet. Nach dem Zeugnis der Apok. selbst hat es ja gerade auch in Kleinasien manchen Gemeinden

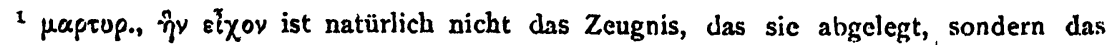
sie empfangen hatten, also der objektive Besitz (Bousset 270) 2war nicht der Glïubigen insgesamt, aber doch der (christlichen) $>M$ ärtyrerk. Auf die lange Reihe von alt.

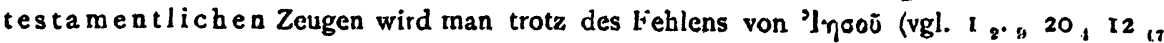
19 10) bei $\mu$ apropiav 6 , doch lieber nicht gleich rekurrieren.

2 Vgl. J. Weiß, Forschungen S. 7 . 
keineswegs an Gelegenheit gefehlt, Geduld und Treue zu bewahren, sie haben den Namen Christi nicht verleugnet 22313 ig 38 10, und

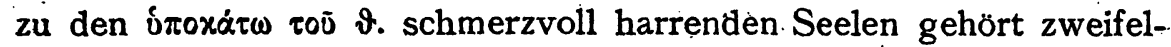
los auch die Seele des pergamenischen Märtyrers Antipas 213.

Wenn IV Esra 435 die in den Vorratskammern harrenden Seelen der Gerechten nach dem Termin ihrer Belohnung fragen, so hat. Boll 33 mit Recht geltend gemacht, daß an dieser wie an den übrigen von Wellhausen (Analyse 10) angeführten Stellen gerade das Eigentümliche der Apok. $6 g_{9}$ erkennbaren Vorstellung (die Seelen, die

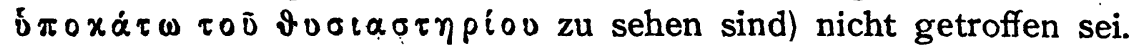

Noch sind diese Seelen nicht im Zustande der Seligkeit, noch sind sie ohne die Kraft und Lebensfülle der Verklärten. Anderseits aber haben wir uns diese nach Rache schreienden Seelen nicht etwa in der Scheol zu denken, dem Lande des Vergessens (Ps $88_{13}$ ), wo man nicht weib, was auf der Oberwelt vor sich geht (Hi 1421; vgl. Eccl 956 10. wo sogar jedes Wissen und Empfinden in der Scheol geleugnet wird; Dalman, Art. »Hades « RE ${ }^{3}$ VII 296). Bildet doch das laute Rufen der Märtyrerseelen einen bemerkenswerten Gegensatz zu dem zirpenden oder wispernden Reden der Schatten im Hades, wie es Jes $8{ }_{19} 29_{4}$ vorausgesetzt wird.

Die Lösung liegt auf einer ganz andern Linie.

Auszugehen ist von der Beobachtung, daß hier e ine Spezialisierung jenes allgemeineren Gedankens vorliegt, indem es sich in diesem Zusammenhang um Seelen einer besonderen Klasse von Gerechten handelt, nämlich von solchen, die eines gewaltsamen Todes gestorben sind.

Unschuldig vergossenes Blut hat Gen 410 eine für Gott vernehmbare, um Rache für die Tat schreiende Stimme; Abel, der erste der Frommen, deren Tod kostbar in Gottes Augen ist Ps $116_{15}$, redet vermöge seines Glaubens noch nach seinem Tode (Hbr i ${ }_{1}$ $12{ }_{24}$ ), sofern sein Blut gen Himmel, also zu Gott (vgl. Lc $15{ }_{18}$ ) schrieund Gott ihn rächte. Allein auch diese alttestamentlichen und urchristlichen Gedankengänge helfen nicht viel zur Aufhellung der Existenz der Märtyrerseelen Apóc $6_{9}$, deren Seligkeit erst eintreten kann, nachdem ihr Blut gerächt ist an den Bewohnern der Erde.

Sehen wir uns daher auf diesem Gebiet der Religionsgeschichte noch etwas weiter um!

Zürnend über den Frevel, also bewußt weiterlebend irrt nach griech is chem ${ }^{1}$ Glauben die Seele des gewaltsam Getöteten, bevor

1 Tert. an. 56. 57 ; Aen. 4, 696 ff. Rohde, Psyche ${ }^{8}$ I 264. 275 f.; II 412.95 .213$. Boll, Offb. S. 32 . 
das ihm geschehene Unrecht 'an dem. Täter gerächt ist, Rache

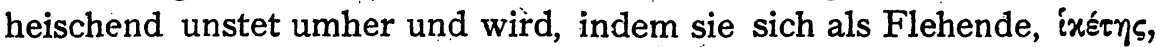

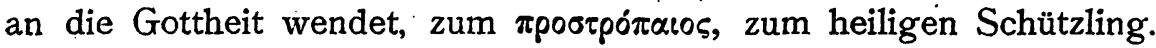
Um Aufnahme in den Hades muß der keine Ruhe findende Braı$\vartheta$ Эóvaros besonders flehen. Nach pythagoreischer, später auch in das populäre Bewußtsein übergegangener Anschauung ist die $\mathrm{Milch}$ -

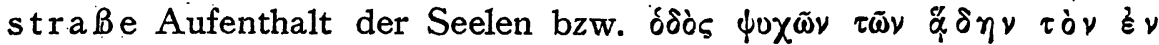

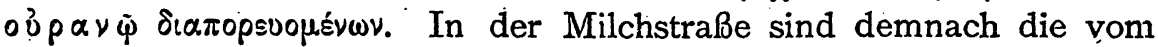

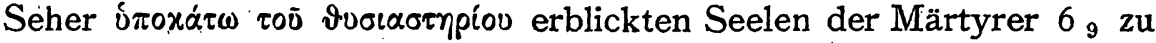
denken, wartend auf die Stunde des Rachevollzuges.

Wie eng diese beiden Ortsbezeichnungen zusammengehören ergibt sich bei der Betrachtung des griechischen Himmelsbildes, das einen Altar in der Milchstraße kennt, und zwar gerade in der Hadesgegend des Himmels ${ }^{1}$. Unter Verweisung auf einen leider verstümmelten Abschnitt der Eratósthenischen Katasterismen ${ }^{2}$ hat Boll gezeigt, daß der Altar am Himmel in der Vision des Apok. der naturgemäße Platz für die Seelen der $\mu \alpha \rho \tau 0 \rho \varepsilon \varsigma$ oi $\pi \iota \sigma \tau \circ i$ (vgl. Apoc $2_{13}$ ) ist, für die Seelen der um des Wortes Gottes und um des $Z$ eugnisses willen, welches sie hatten, Geschlachteten $6_{9}$, deren Blut von den Gott feindlichen Bewohnern der Erde vergossen ist 610 .

Glühend bricht das Rachebegehren der - wohl in sitzender ${ }^{3}$

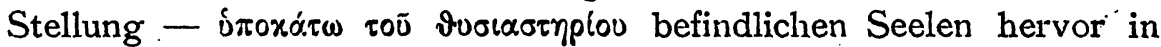
dem leidenschaftlichen, mit lauter ${ }^{4}$ Stimme an Gott, den heiligen und wahrhaftigen Herrn ${ }^{5}$, gerichteten Gebet $6_{10}$ : "Bis wielange wirst $d u$ nicht richten und rächen unser Blut an den Bewohnern der Erde?

1 Boll, Offb. 34. 72. 74. 88, wo darauf hingewiesen wird, daß sich an die Erwähnung der Todesregion des Himmels Apoc 6. 7. 8 die Seelen der Märtyrer 6 , aller-

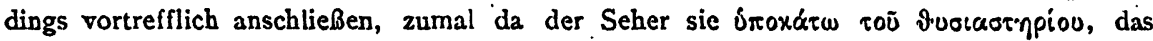
ebendort lokalisiert ist, erblickt. Dieser Zusammenbang entspricht der eingangs erwähnten Beobachtung, daß sich der Apok. hier an den Gedankengang von Sach I an-

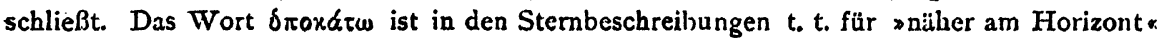
(Boll, Sphära S. 89, 1).

2 ed. Robert p. 182 ; Boll, Offb. S. 33 f. 74.

${ }^{3}$ Sitzen als Zeichen der Hilflosigkeit bei Bittflehenden Thuc. 1, 24, 7 im Tempel der Hera; am Altar der Athena Polias I, 126, 10; auf dem Hausherd I, 136, 3; 137, 1 : Zuflucht bei den Altären 3, 28, 2; Schutz suchend in den Tempeln 3, 70, 5 ; im Ternpel der Hera 3, 75, 5; im Tempel der Dioskuren 3, 75, 3 .

4. S. S. 233 .

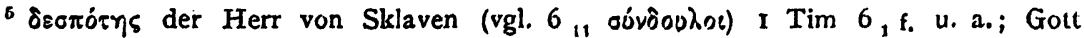
Ic $2_{29}$, Act 4 24, Christus 2 Pt 2 , Judac 4, LXX für אלדים 
Die Vermutung liegt nahe, daß auch dies Rachegebet der $\delta$ i 0 -

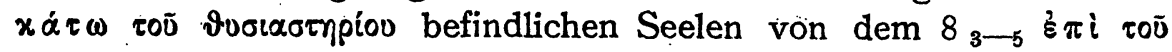

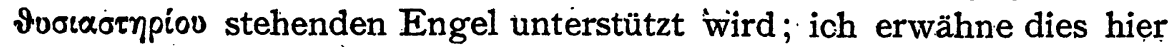
aber nur darum, weil man sich in diesem Falle auch den Altar 6, als $R$ äucheraltar vorzustellen hätte ${ }^{1}$.

Die harrenden Märtyrerseelen erhalten zunächst einen vorläufigen Bescheid, nämlich die mit einer symbolischen Handlung verbundenen Anweisung 6 ${ }_{11}$. Das weibe, also die Anwartschaft auf Teilnahme an einem bevorstehenden Freudenfest (vgl. Mt 22 12) darstellende, für den Festsaal passende Gewand - »in dieser Hinsicht war das orientalische Zeremoniell besonders empfindlich " 2 ist eine $\sigma \tau 0 \lambda \eta$, also ein faltiger, bis auf die Füße herabreichender kostbarer Talar, wie bei der unzählbaren Schar vor Gottes Thron

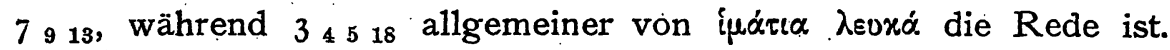
- Nach dieser die Gewißheit der Realisierung ihrer Erwartung verbürgenden Überreichung des Kleides erfolgt die ausdrückliche, aber ebenfalls nur erst vorläufige, Antwort auf das 》Wann?《 Es wird nicht

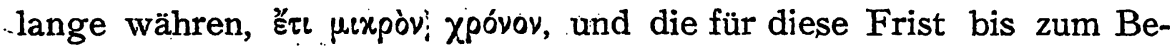
ginn des Freudenfestes geltende, für $\beta$ เ

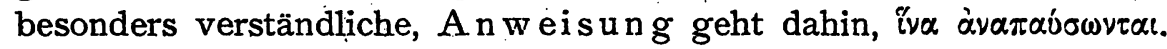
Sie sollen offenbar der trostreichen Verheißung gedenken, mit der Daniel nach den Aufschlüssen über die Endzeit von dem Engel entlassen wird: "Du wirst ruhen und auferstehen $z u$ deinem Lose (vgl. Col $\mathrm{I}_{12}$ ) am-Ende der Tage $\ll$, Dan $12{ }_{13}{ }^{3}$.

Die àyó $\pi \alpha u o t s$ soll in dem Moment ihr Ende finden, wenn auch

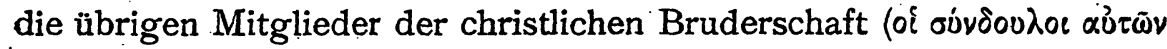
xaì of $\alpha \delta \varepsilon \lambda$ yoi $\alpha \dot{0} \tau \tilde{\omega} y)$, soweit sie zum Martyrium bestimmt sind, eben dies ihr Martyrium ws xaì aưoi, die $\sigma_{g}$ bereits im Besitz der $\mu \alpha \rho-$ topia sind, vollendet haben werden; das $\varepsilon^{\prime} \omega \varsigma_{11}$ entspricht genau dem

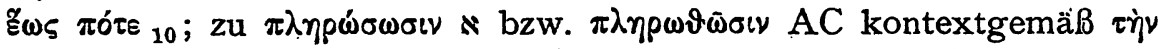

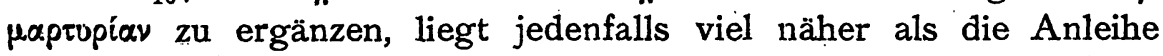
bei IV Esra 436 f., Apoc Bar $30_{2}$ (am allerwenigsten gehört Mt 2332 hierher) wonach an die Vollendung der $\mathrm{Zah} 1$ der Märtyrer zu denken

1 Nach Boll, Sphära 148; wurde das griechische Sternbild des Altars als Opfertisch oder als RauchfaB dargestellt. Ganz ohne Grund will man (im Unterschied von

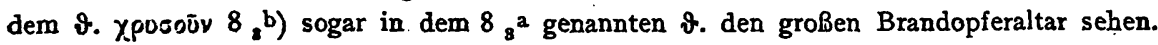

2 Jülicher, Gleichnisreden Jesu III S. 424. Uber die der Mysteriensprache angehörigen verborgenen Lichtgewänder vgl. Böll, Offb. S. 33. 35. 49, I (100, 5).

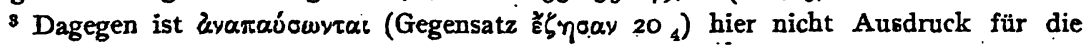
selige himmlische Ruhe; zu der wegen $4_{8}$ beliebten Deutung, sie sollten aufhören, um

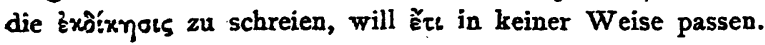


wäre . Mag also der Aufschub des Gerichtsvollzuges sonst in der "Apokalyptik in ähnlicher Weise erklärt werden (Holtzmann 447), in keiner von den beigebrachten. Parallelen braucht der Begriff "Zahl* erst (wie hier) ergänzt zu werden, und nirgendwo ist (wie hier) von Märtyrern die Rede.

II

»Bald ist die Wartezeit entflohn.» Über diese unbestimmte Formulierung kommt der vorläufige Bescheid nicht hinaus. Wo aber schildert die Apoc die def in itive Erhörung des Rachegebets

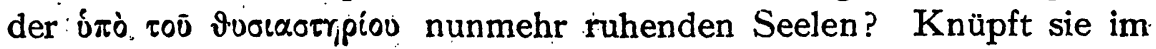
weiteren Verlauf der Handlung überhaupt an die fünfte Siegelvision inhaltlich noch an, oder hat man in diesem seltsamen Bild bloß ein eingesprengtes Fragment $\mathrm{zu}$ sehen, ohne charakteristische Bedeutung für das Ganze?

Trotz der stereotypen Ausdrucksweise des Apok. kann es unmöglich rein auf Zufall beruhen, wenn die Einführungsformel der Vision $6_{9}$ :

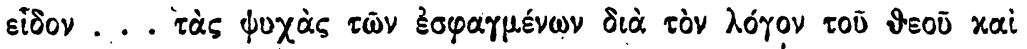

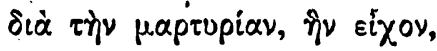

gerade an der entscheidenden Stelle $2 \mathrm{O}_{4}$ mit unwesentlichen Abweichungen wiederholt wird:

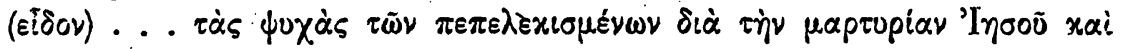

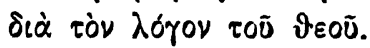

Sehen wir näher $z u$, unter welchen besonderen Umständen diese Wiederaufnahme des Wortlauts erfolgt! Babel ist gefallen $\left(1_{1}{ }_{1}\right.$ bis $\left.18{ }_{24}\right)$, und nach ihrem Sturz findet sich in der Weltstadt $18{ }_{20} \rightarrow 24$ Blut von Propheten, Aposteln und Heiligen und aller, die hingeschlachtet wurden auf Erden. Diese an $17_{6}$ anknüpfende Erwähnung der Christenmorde gewinnt aber noch an Bedeutung, wenn man sie, wie der Zusammenhang es fordert, als Einleitung zur Wiederaufnahme und Durchführung des inzwischen mehrfach leise erklungenen Märtyrerblut-Motivs $66_{9 \rightarrow 11}$ auffaßt ${ }^{2}$.

- 1 Bousset 272. Bezeichnenderweise will Bousset xai nach $\pi$ i. $\eta p \omega \vartheta \tilde{\omega} e \iota \nu$ tilgen; dies xai stimmt zu der Auffassung Boussets in der Tat so wenig wie zudem Vorschlag

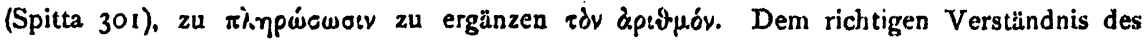
$\pi \lambda$ inpúswoev kommt es schon nüber, wenn man nach Analogic von Act $20_{20}$. II Tim 4, erklärt sden Lauf vollenden

2 Das Blut der auf Erden ( 18 24) hingeschlachteten Märtyrer ist also nichl wie ihre Seeles unter dem Altar 6, zu suchen; die Vorstellung, daß in dem Blut der Opfertiere Lev 4 , die Seele wohnt, kommt Apoc $6_{10}$ so wenig in Betracht wie die Tatsache, daB das Opferblut Lev 4 , unten am Brandopferaltar abfließt. Auch die weitere. Vor- 
Ob die Stimme aus den vier Hörnern des Altars von Gold 9 18. als Antwort auf die Bitte $8{ }_{3 \text { f. }}$ gelten (Bousset 302 ) und òb ${ }_{14}{ }_{18}$ an den Brand opferaltar (Bousset 389) gedacht werden darf, oder ob vielmehr an beiden Stellen eine nähere Beziehung zu der Szene $6{ }_{9} 11$ anzunehmen ist, kann vorläufig unerörtert bleiben. Um so wichtiger ist für uns eine durch die, unmittelbare Nähe der Babylonvision nur noch bedeutsamere charakteristische Episode, die - gleichsam ein Januskopf - rückwärts auf die Szene $6{ }_{9-11}$. vorwärts auf die Vision $19_{1}-20_{4}$ blickt und, falls sie vom Herausgeber dazu ausersehen wäre, zwischen beiden Abschnitten eine Verbindung herzustellen, ihren Zweck ausgezeichnet erfüllen würde. Nachdem nämlich der dritte Schalenengel $16{ }_{4}$ die Flüsse und Wasserquellen, also das Trinkwasser, in Blut verwandelt hat - eine angemessene Strafe ("sie sind es wert" $16_{6}$ ) für das Vergießen von Märtyrerblut - gibt (innerhalb des Heiligtums, aus dem 156 die Schalenengel hervorgehen) $16_{7}$ bezeichnenderweise »der Altar" seiner Genugtuung über diese Vergeltungsmaßnahmen Ausdruck: "Wahrlich, Herr, Gott, Allbeherrscher, wahrhaftig und gerecht sind deine Gerichte« - Worte, die unmittelbar an die leidenschaftlich erwartungsvolle Frage der íjo-

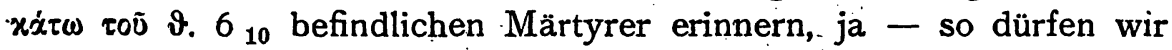
schließen - gewiß auch erinnern sollen.

Die $18{ }_{24}$ aufgezählten Kategorien von Christen, deren Blut in Babel gefunden wird, werden $17{ }_{6}$ als Zeugen Jesu « zusammengefaßt: es sind die um (des Wortes Gottes und des Zeugnisses willen, welches sie hatten, Geschlachteten $6{ }_{9} 20_{4}$. Die besondere Erwähnung der Propheten erklärt sich aus der erläuternden Bemerkung 19 10: das Zeugnis Jesu ist der Geist der Prophetie, ein Satz, der freilich von den meisten Kritikern als "ungeschickt eingeschobene Glosse « beseitigt wird. Bedarf aber nach Ansicht des. A pok. dieser Teil der Formel einer ausdrücklichen Erklärung, so wird man fragen,

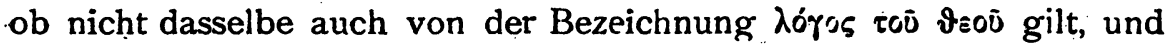

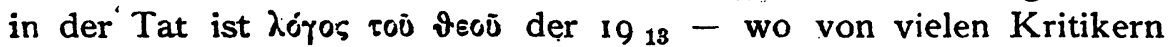
konsequenterweise der »müßige Einfall eine Abschreibers" konstatiert wird - enthüllte Name des blutbesprengten Reiters >Treu und (vgl. $6_{10}$ ) Wahrhaftig* auf weißem Roß, der mit Gerechtig-

schrift der Thorah, daß das Blut der Sündopfer nicht bloß an den Brandoṕferaltar, sondern auch an die Hörner (vgl Apoc $9_{13}$ ) des Rauchopferaltars $k \cdot m$ (Lev $4,{ }_{18} 16{ }_{18}$ ), wäre demnach für die Erklärung von Apc $6,{ }_{10}$ bedeutungslos. Keinesweus tritt hier eben das Martyrium sunter den Gesichtspunkt eines Gott wohlgefälligen Opfers \& (Holızmann 446), so daß die Mărtvrer als »Gott geweihte Opfer« (Bousset 26,) charakterisiert wäıen, eine Berufang auf $\mathrm{Phl}{ }_{17}$, II Tım 4 , kạnn nur verwirrend wirken. 
keit richtet. (vgl. $6_{10}$ ) und kämpft. $Z$ u seiner Beschämung muß der Leser hier gestehen, daß er die gedankenschweren Andeutungen $6_{10}$ in ihrem Vollsinn nicht erfaßt, ja sogar als solche vielleicht nicht einmal erkannt hat! Aber noch etwas anderes wird in diesem $\mathrm{Zu}$ sammenhang erklärt: die Lichtgewänder (vgl. $3 \ddot{5} \quad 7$ g), die $6_{11}$ den Märtyrerseelen als "Symbol der Erhöhung zu den lichten Regionen " (Boll, Offb. S. 49) gereicht werden, sind nach Analogie des strahlenden, reinen Byssuskleides, mit dem sich das. Weib des Lammes zur Hochzeit geschmückt hat, die Gerechtigkeit der Heiligen $19 \mathrm{~s}$ eine Angabe, die Bousset 428 allerdings wiederum als recht nichtssagende Zutat eines müßigen Abschreibers《 ansehen möchte.

Wer jene Erläuterungen $19 \mathrm{~s}_{1013}$ als ästhetische Anstöße empfindet und infolgedessen als störende, unorganische Zutaten einer unberufenen Hand ohne weiteres ausscheidet, wird der schriftstellerischen Eigenart des Apok. letzter Hand nicht gerecht. Solche überraschenden Wendungen gehören zum apokalyptischen Stil, der Mann aus dem Volk empfindet sie als geistreich, und die angeblichen Glossen im Text unserer Apok. hätten wohl eine besondere, zusammenhängende Untersuchung verdient; sie würde wahrscheinlich ergeben, daß bei der Mehrzahl dieser scheinbar unorganischen Einschaltungen eine in mehrfacher Hinsicht einheitliche Methode nicht $z u$ verkennen ist. Bei dieser Gelegenheit sei noch einmal ausdrücklich daran erinnert. daß die vorliegenden Studien es mit der Johannesapoc in ihrer gegenwärtigen Gestalt zu tun haben, nicht mit einer ursprünglicheren Fassung oder mit etwa zugrunde liegenden Quellenschriften.

Auf den Gang der Handlung gesehen, ist es - zumal angesichts der $2 \mathrm{O}_{4}$ vorliegenden Verwertung der Einführungsformel $6_{9}$ - durchaus nicht unmöglich, daß die Notiz $6_{11}$ "es ward ihnen (den Seelen der Märtyrer) gegeben ein weißes Kleid" in der Wendung $19_{8}$ "es ward ihr (dem Weib des Lammes) gegeben, sich anzutun mit strahlendem, reinem Byssus mit Bewußtsein fast wörtlich wieder aufgenommen ist. Dementsprechend wird der Seligerklärung der zum Hochzeitsmahl des Lammes Geladenen I 9 g hier eine besondere Bedeutung zukommen: diese Seligerklürung ist innerhalb des Aufbaues der Apoc nichts Geringeres als die Erfüllung der Anwartschaft $6_{11}$, die Einlosung des symbolischen Bestandteils jener Antwort, und es ist wahrlich kein bloßer Zufall, wenn in unmittelbarem Anschluß daran 1910 der angelus interpres die auf jenes herrliche Verheißungs- 
wort hin vom Seher beabsichtigte Anbetung. mit einer gerade an die Märtyrerszene des fünften Siegels mehrfach kräftig anklingenden Motivierung abwehrt ${ }^{1}$ : er sei nichts weiter als Mitknecht $\left(6_{11}\right)$ des Sehers und seiner Brüder $\left(6_{11}\right)$, die das Zeügnis $\left(6_{9}\right)$. Jesu haben $\left(6_{9}\right)$. Daraus folgt, daß sich die Anfrage der Märtyrer $6{ }_{9}$ nach ihrer im Wortlaut unmittelbar gegebenen negativen Seite als mit der Abšăge der triumphierenden Stimme. I9:2 ("wo das Begehren der Märtyrer fast wörtlich in einen Lobgesang umgesetzt wird «, Bengel) erledigt ansehen läßt. Allein das Racheverlangen der Märtyrerseelen ist - wie wir uns überzeugt haben - nicht Selbstzweck, sondern die conditio sine qua non für die Beendigung ihres Aufent-

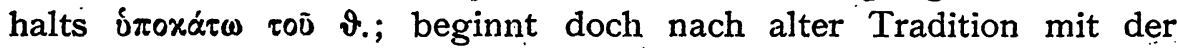

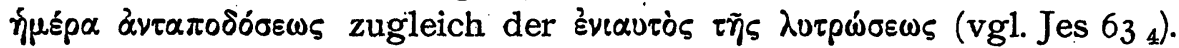
Was liegt näher als die Annahme, daß dem Apok. die Situation $6{ }_{9}$ auch $2 \mathrm{O}_{4}$ vorschwebt beim Anblick der Seelen der um des Zeugnisses Jesu und um des Wortes Gottes willen Enthaupteten, die dann als Teilnehmer am Millenium charakterisiert werden:

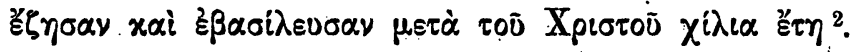

$\mathrm{Daß}$ das »tausendjährige Reich « nịcht bloß vorzugsweise $(\mathrm{Htzm}$ 493), sondern ausschließlich für die Märtyrer bestimmt ist, hat man bei der exegetischen Fundamentierung wie in den historisch-kritischen Darstellungen des Chiliasmus bisher nicht genügend beachtet. Manche wilde Spekulation würde unterblieben sein, wenn die Tatsache mehr zu ihrem Recht gekommen wäre, daß im Sinne des Apok., abgesehen von den Märtyrern alle übrigen, also auch die eines ruhigen Todes sterbenden, Gläubigen an der ersten Auferstehung und an der Königsherrschaft und Freude des Milleniums nicht teilhaben. Dabei ist es von untergeordneter Bedeutung, ob die in der Verfolgung des Tieres standhaft Gebliebenen $2 \mathrm{O}_{4} \mathrm{~b}$ neben den eigentlichen Märtyrern eine besondere Klasse bilden können (Bousset 437) oder - als die II 7 I3 15 dafür den

1 Die Formel »Bete Gott an! \& bedeutet fast dasselbe wie die Mahnung Deut 6 , die Budde (Auf dem Wege zum Monotheismus, 1910, S. 18) treffend umschreibt: >Wir brauchen keine Vertreter und. Statthalter, keine Vermittler und. Fürsprecher: zu Jahwe selber dürfen wir uns nahen und sicher sein, daß er uns Gehör schenkta. Der Seher soll in dem Engel lediglich ein Geschöpf Gottes und damit seines gleichen (vgl. Budde S. 21) erkennen. Man spürt hier etwas von dem im Kolosserbrief hervortretenden Gegensatz zum Engelkultus und Gestirndienst, auf dessen Bedeutung innerhalb des Weltbildes der Apoc. Boll so nachdrücklich aufmerksam gemacht hat.

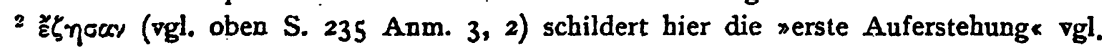
$20_{5}$ e $14_{14} 21_{8} 2_{11}$ LC $\mathrm{I}_{14}$; es besagt also, daß die Seelen der Märtyrer nunmehr mit dem für sie bestimmten himmlischen Leib ausgestattet werden. 
Tod hatten erleiden müssen - mit ihnen identisch sind (Htzm 492). Schwerlich aber wird man sich auf diese Stelle berufen wollen als Beleg für die oben bereits als unwahrscheinlich erwiesene Deutung des $\pi \lambda \eta p \omega ́ \sigma \omega \sigma \iota v 6_{11}$ : es bleibt dabei, daß dort nicht an die Auffüllung der Gesamtzahl der zum Martyrium Bestimmten, sondern

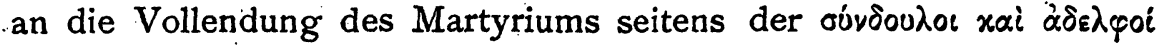
gedacht ist.

Den endgültigen, nicht mehr blob in Worten und Symbolen bestehenden Bescheid erhalten die Märtyrerseelen also mit dem Anbruch des Milleniums. Dann ist ihr Blut gerächt an den Bewohnern der Erde, und gleich ihren Mitknechten und Brüdern, die ebenfalls ihr Martyrium vollendet haben, haben sie kraft ihres weißen Kleides Zutritt zum Hochzeitsmahl des Lammes. Das ist die Erhörung des Gebets der Märtyrerseelen im Sinne des Apok. letzter Hand.

Jedenfalls ist uns die Annahme; daß die beiden Abschnitte $66_{9-11}$ und $19_{1}-20_{6}$ im Sinne des Apok. letzter Hand aufs engste zusammengehören, zum Schlüssel für eine Reihe von bisher ungelösten Schwierigkeiten geworden. Je mehr sie an Wahrscheinlichkeit gewinnt, in desto helleres Licht tritt der Gedankenfortschritt der Apoc, desto mehr beginnen die Fäden der verschiedenen Bestandteile des künstlichen Gewebes sich zu entwirren. Erhebt sich nun die Frage, ob diese beiden einander ergänzenden Stücke, das Gebet der Märtyrerseelen und seine Erhörung, ursprünglich in einer besonderen $Q$ uellenschrift des Apok. unmittelbar oder doch näher beieinander gestanden haben, so ist eine weitere kritische Analyse der in unserer Johannesapoc zwischen beiden Perikopen liegenden Szenen und eine genaue Untersuchung ihres Verhältnisses zum Ganzen des dramatischen Aufbaues erforderlich, die bei d.m Resultat dieser Studie einzusetzen hätte. Hier sollte nur die Bedeutung der beiden in ihrer inneren Struktur von Sacliarja beeinflußten Abschnitte für die Schrift des Endredaktors nachgrewi'sen werden: das Gebet der Märtyrerseelen weiß er zu einem crregenclen Moment von hervorragender Wichtixkeit auszuyestalten, die Erhörung des Gebets wird ihm zu einem integrierenden Bestandteil der dramatischen Katastrophe.

[Abgeschlossen am 1. Oktober 1921.] 